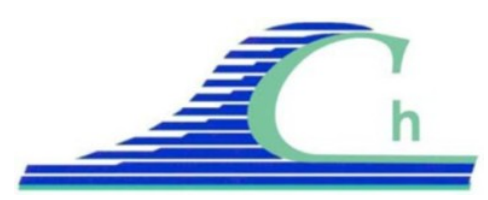

\title{
Présentation des critères de conception du port Tanger Med II
}

\section{Moussa BENAMAR $^{1}$, Said AMAKHLOUF ${ }^{1}$}

1. Société Tanger Med Engineering (TME), Zone franche Ksar Al majaz, Tanger, Maroc.

m.benamar@tme.ma;m.amakhlouf@tme.ma

\section{Résumé :}

Le nouveau port Tanger-Med II est constitué principalement d'un quai de $2800 \mathrm{ml}$ à $-16 \mathrm{~m} \mathrm{ZH}$, desservant un terre-plein arrière de $150 \mathrm{Ha}$ de superficie, ainsi que d'un poste pétrolier. Les ouvrages de protection sont constitués d'une digue principale de $3875 \mathrm{ml}$ dont $955 \mathrm{ml}$ de digue à talus et $2920 \mathrm{ml}$ de digue à caissons (96 caissons), et d'une digue secondaire à talus de $1163 \mathrm{ml}$ avec un tenon constitué de 6 caissons.

Sur la base d'un canevas délimitant la zone de développement du port en termes de coordonnées, un plan de masse devra être défini de façon à respecter les exigences de fonctionnalités fixées pour chacune de parties constituant le port.

Pour les voies navigables :

- Dimension, position et profondeur du chenal d'accès, de la zone d'évitage et du bassin du port.

- Recouvrement des deux ouvrages de protection principaux.

Pour les quais à conteneurs, et vrac liquide :

- Caractéristiques en termes de linéaire, de profondeur, de la cote d'arase et de l'étendu de la zone de mise à poste ainsi que les caractéristiques des navires qui y seront accostés.

- Tassements admissibles du mur de quai.

Pour les ouvrages de protection :

- Franchissements admissibles.

- Taux d'indisponibilité des navires à quai.

- Stabilité des ouvrages sous l'effet des houles centennales, du séisme etc.

- Traficabilité de la dalle de circulation longeant les ouvrages de protection.

- Protection des infrastructures des conduites hydrocarbures et équipements de postes pétroliers.

Pour les terre-pleins :

- Cote d'arase et pente pour le drainage des eaux pluviales.

- Tassements admissibles.

- Compacité des terre-pleins (densité relative, résistance de pointe minimale mesurée au CPT, ...).

Pour répondre à l'exigence de durabilité des ouvrages correspondant à une durée de vie minimale de 100 ans, les exigences suivantes ont été définies : 
- Les ouvrages en béton armé seront confectionnés avec un BHP.

- Tous les bétons armés seront munis d'une protection cathodique.

- Une limitation au niveau de la pénétrabilité des ions chlorures dans le béton.

- Caractéristiques des bétons non armés : dosage et type de ciment, etc.

Mots clés :

Port - Portuaire - Maritime - Contrat - Critères - Exigences - Conception Fonctionnalité - Dimensionnement - Tanger Med 2

\section{Introduction}

Le but de la communication serait de présenter les critères de conception du projet de construction du nouveau port Tanger-Med II. Le port est constitué principalement d'un quai de $2800 \mathrm{ml}$ à $-16 \mathrm{~m} \mathrm{ZH}$, desservant un terre-plein arrière de $155 \mathrm{Ha}$ de superficie, ainsi que d'un poste pétrolier. Les ouvrages de protection sont constitués d'une digue principale de $3665 \mathrm{ml}$ dont $955 \mathrm{ml}$ de digue à talus et $2710 \mathrm{ml}$ de digue à caissons $(96$ caissons), et d'une digue secondaire à talus de $953 \mathrm{ml}$ avec un tenon constitué de 6 caissons.

\section{Exigences de fonctionnalités}

Sur la base d'un canevas délimitant la zone de développement du port en termes de coordonnées, un plan de masse devra être défini de façon à respecter les exigences de fonctionnalités fixées pour chacune de parties constituant le port.

\subsection{Les voies navigables}

\subsubsection{Caractéristique de la darse à conteneurs :}

Les darses conteneurs doivent être sans angles pouvant gêner les manœuvres et l'accostage des navires aux différents postes.

- Largeurs darse (entre le pied de la digue principale et le pied du quai à conteneurs d'autre part) : côté Ouest $250 \mathrm{~m}$ à l'origine du quai D1, et $400 \mathrm{~m}$ à son extrémité. Pour l'implantation des postes pétroliers, il faudra veiller qu'entre les limites de navigation, localisées à $70 \mathrm{~m}$ du quai conteneurs et à $50 \mathrm{~m}$ de la coque des pétroliers, une largeur utile minimale de navigation de $240 \mathrm{~m}$ doit être atteinte.

- Largeurs darse (entre le pied de la digue secondaire et la magistrale du quai) : côté Est de $300 \mathrm{~m}$ à l'origine du quai $\mathrm{C} 1$ et $350 \mathrm{~m}$ à son extrémité ouest et $365 \mathrm{~m}$ à l'extrémité du quai $\mathrm{C} 2$ après extension éventuelle de la digue secondaire par Le Maître d'ouvrage à terme.

\subsubsection{Dimensions et positions du chenal d'accès et de la zone d'évitage.}

Le chenal d'accès et la passe d'entrée du Port : Il doit être implanté et orienté de façon à éviter les interférences avec le chenal d'accès du Terminal Spécialisé Roulier, à garder 


\section{XII ${ }^{\text {èmes }}$ Journées Nationales Génie Côtier - Génie Civil \\ Cherbourg, 12-14 juin 2012}

un angle et une distance de sécurité convenable par rapport à la digue secondaire, à permettre un arrêt des navires en toute sécurité et à limiter la pénétration des houles. La largeur minimale de la passe d'entrée et du chenal prise à $-20 \mathrm{~m}$ ZH est de $330 \mathrm{~m}$. Les profondeurs au niveau du chenal d'accès doivent être de $-19 \mathrm{~m}$ ZH.

La zone d'évitage doit satisfaire les conditions suivantes :

- L'ellipse d'évitage doit avoir les dimensions de $900 \mathrm{~m} \times 650 \mathrm{~m}$.

- Son centre doit être à une distance minimale de $950 \mathrm{~m}$ par rapport à la passe d'entrée.

- Elle doit être centrée par rapport au quai à conteneurs quand celui-ci est réalisé dans sa totalité sur $2800 \mathrm{~m}$.

- Les cotes du fond doivent être de -17 m ZH.

\subsubsection{Recouvrement des deux ouvrages de protection principaux :}

Le recouvrement des deux ouvrages de protection principaux (digue principale et digue secondaire) compté parallèlement à la direction du chenal d'accès doit être sur une longueur d'au moins $375 \mathrm{ml}$. Néanmoins, le Maître d'Ouvrage peut accepter un recouvrement légèrement inférieur dans la mesure où les critères de fonctionnalité du présent document sont vérifiés. Ce paragraphe s'applique uniquement en Phase 2 (hors Base-Phase1).

\subsubsection{Profondeurs du chenal d'accès au port et du bassin du port :}

Les profondeurs au niveau du chenal d'accès doivent être de $-19 \mathrm{~m}$ ZH. Les profondeurs du bassin du port sont :

- A l'ouest de la zone d'évitage : : $-16.5 \mathrm{~m} \mathrm{ZH}$

- A l'est de la zone d'évitage : : $-16.0 \mathrm{~m} \mathrm{ZH}$

\subsection{Les quais à conteneurs, et vrac liquide}

\subsubsection{Navire de projet :}

Les navires de projet à considérer pour la conception des ouvrages sont ceux définis dans le tableau 1.

Tableau 1.

\begin{tabular}{llllll}
\hline Type & $\begin{array}{l}\text { Port en lourd } \\
(t)\end{array}$ & $\begin{array}{l}\text { Déplacement } \\
(t)\end{array}$ & LOA $(\mathrm{m})$ & Largeur $(\mathrm{m})$ & $\begin{array}{l}\text { Tirant d'eau } \\
(\mathrm{m})\end{array}$ \\
\hline Grands & 140000 & 205000 & 350 & 46 & 15 \\
Porte-conteneurs & 156000 & 240000 & 395 & 56 & 15,5 \\
\hdashline Feeder & 27000 & 36000 & 200 & 30 & 11,5 \\
\hdashline Petrolier & 150000 & 184000 & 284 & 50 & 17 \\
& 20000 & 30000 & 180 & 26 & 10 \\
\hline
\end{tabular}




\subsubsection{Caractéristiques géométriques :}

Pour le quai à conteneurs, elles se présentent comme suit :

- Un linéaire de $2800 \mathrm{ml}$ rectiligne.

- Une profondeur de $-16 \mathrm{~m} \mathrm{ZH}$.

- Une cote d'arase est à $+4.50 \mathrm{~m} Z \mathrm{ZH}$.

- Une zone de mise à poste devant le quai de $70 \mathrm{~m}$ de large, et de profondeur atteignant les $-16 \mathrm{~m} \mathrm{ZH.}$

Pour le quai dédié aux vrac liquides :

- Un linéaire de $112 \mathrm{ml}$ pour chacun des deux postes.

- Une cote d'arase à $+4.50 \mathrm{~m} \mathrm{ZH}$.

- Une profondeur de -18 m ZH et -17 m ZH respectivement pour les deux postes situés le long de la digue principale.

- La distance de sécurité autour du navire à poste par rapport aux voies navigables est de $50 \mathrm{~m}$.

- Pour le premier poste qui est fondé à $-18 \mathrm{~m} \mathrm{ZH}$ : Une zone de mise à poste devant le quai de $60 \mathrm{~m}$ de large et $300 \mathrm{ml}$ de long à draguer à $-18 \mathrm{~m} \mathrm{ZH}$.

- Pour le second poste pétrolier qui est fondé à $-17 \mathrm{~m} \mathrm{ZH}$ : Une zone de mise à poste, sera dragué à $-17 \mathrm{~m}$ ZH sur $50 \mathrm{ml}$ de large et $250 \mathrm{ml}$ de long.

\subsubsection{Tassements admissibles du mur de quai :}

Les tassements admissibles maximaux au niveau du mur de quai seront inférieurs aux valeurs suivantes :

- $25 \mathrm{~mm}$ au bout de 1 an.

- $40 \mathrm{~mm}$ au bout de 5 ans.

- $60 \mathrm{~mm}$ au bout de 20 ans.

\subsection{Les ouvrages de protection}

\subsubsection{Franchissements admissibles}

Les critères définis pour les franchissements des digues sont les suivants :

Pour les parties de digues avec terre plein et/ou conduites hydrocarbures et/ou poste à l'arrière :

- Débit de franchissement mesuré à la crête de la digue (mur de couronnement le plus proche du bassin) $\leq 1 \mathrm{l} / \mathrm{m} / \mathrm{s}$ pour des conditions de houle décennales associées à un niveau d'eau haut.

- Le critère de stabilité d'un éventuel talus arrière doit être respecté pour des conditions de houle centennales associées à un niveau d'eau haut.

Pour les musoirs et parties de digues sans terre-plein, poste ou conduites en arrière :

- Débit de franchissement au droit du mur de couronnement le plus proche du bassin $\leq$ $30 \mathrm{l} / \mathrm{m} / \mathrm{s}$ pour des conditions de houle décennale associées à un niveau d'eau haut. 


\section{XII ${ }^{\text {èmes }}$ Journées Nationales Génie Côtier - Génie Civil \\ Cherbourg, 12-14 juin 2012}

- Le critère de stabilité d'un éventuel talus arrière doit être respecté pour des conditions de houle centennales associées à un niveau d'eau haut.

- L'impact des franchissements devra être intégré dans les études d'agitation du bassin. La digue sera conçue de manière à ce que les futurs équipements (conduites, équipements des postes pétroliers, ...) ne soient pas endommagés pour des conditions de houle centennales.

\subsubsection{Taux d'indisponibilité des navires à quai.}

Critère d'agitation devant chaque poste - Navires à poste :

- Postes pour porte-conteneurs : houle incidente résiduelle créée par l'ensemble des houles : $0.7 \mathrm{~m}$ dépassée 1\% du temps (cette hauteur admissible pouvant être plus élevée pour les houles d'Est sous réserve de justification sur la base de calculs de tenue de navires à poste pour le porte conteneur de $350 \mathrm{~m}$ et en se limitant à $100 \%$ d'opérabilité).

- Postes pour porte-conteneurs : houle incidente résiduelle créée par l'ensemble des houles : $0.6 \mathrm{~m}$ dépassée $2 \%$ du temps (cette hauteur admissible pouvant être plus élevée pour les houles d'Est sous réserve de justification sur la base de calculs de tenue de navires à poste pour un feeder de $200 \mathrm{~m}$ et en se limitant à $75 \%$ d'opérabilité).

- Postes pour porte-conteneurs : houle incidente résiduelle créée par l'ensemble des houles : $0.5 \mathrm{~m}$ dépassée environ 3\% du temps (cette hauteur admissible pouvant être plus élevée pour les houles d'Est sous réserve de justification sur la base de calculs de tenue de navires à poste pour un feeder de $150 \mathrm{~m}$ et en se limitant à $75 \%$ d'opérabilité).

- Postes pétroliers : houle incidente résiduelle créée par l'ensemble des houles : $0.7 \mathrm{~m}$ dépassée $5 \%$ du temps.

Mouvements de référence - Navires à postes : Les valeurs (PIANC, 1995) de référence pour l'analyse des mouvements au centre de gravité des navires conteneurs sont présentés dans le tableau 2.

Tableau 2.

\begin{tabular}{lll}
\hline Mouvements & $\begin{array}{l}\text { Valeur admissible pour 100\% } \\
\text { d'opérabilité }\end{array}$ & $\begin{array}{l}\text { Valeur admissible pour 50\% } \\
\text { d'opérabilité }\end{array}$ \\
\hline Cavalement (m) & 1.0 & 2.0 \\
Embardée (m) & 0.6 & 1.2 \\
Pilonnement (m) & 0.8 & 1.2 \\
Roulis (deg) & 3.0 & 6.0 \\
Tangage (deg) & 1.0 & 2.0 \\
Lacet (deg) & 1.0 & 1.5 \\
\hline
\end{tabular}


Houle et vents - critères minimaux retenus pour la manœuvrabilité des navires en évolution :

- Entrée et accostage : Courant max de vives eaux moyennes, Houle de secteur Ouest et Est dépassée $1 \%$ du temps et Vents de $12 \mathrm{~m} / \mathrm{s}$.

- Appareillage et sortie : Courant max de vives eaux moyennes, Houle de secteur Ouest et Est dépassées 1\% du temps et Vents de $15 \mathrm{~m} / \mathrm{s}$.

Sauf accord du Maître d'Ouvrage, la concomitance houle-vent ne sera pas prise en compte pour l'évaluation des taux d'indisponibilité des postes. Ces études doivent analyser :

- les taux d'agitation devant les quais, les zones du bassin et les zones de manœuvre ;

- les conditions de tenue à poste des navires de projet avec plan et conditions d'amarrage ;

- les taux d'indisponibilité des différents postes ;

- les conditions d'entrée et d'accostage aux différents postes ;

- les conditions d'appareillage et de sortie des navires ;

- les taux d'indisponibilité des accès et des zones de manœuvre du port.

La validation du plan de masse du point de vue manœuvrabilité a été faite par la commission nautique du complexe portuaire TANGER MED composée de :

- le commandant du port

- le pilote majeur

- un pilote confirmé

- un pilote expert choisi par le maître d'ouvrage

La commission définira le programme des essais de manœuvrabilités et participera à la réalisation de toutes les manœuvres.

Toute manœuvre réussite du premier coup est validée.

Toute manœuvre négative doit être refaite par deux autres pilotes de la commission, la manœuvre sera réputée négative si elle n'est pas réussie deux fois sur trois essais.

\subsubsection{Stabilité des ouvrages sous l'effet des houles :}

Il se distingue nettement deux climats, un d'Ouest provenant de l'Atlantique et un d'Est provenant de la Méditerranée.

Par ailleurs, les houles interviennent dans deux types de problématiques :

- les houles opérationnelles, définies par leurs statistiques d'occurrence, impactent l'agitation dans le port

- les houles extrêmes interviennent dans le dimensionnement des ouvrages (franchissements et stabilité) 


\section{XII ${ }^{\text {èmes }}$ Journées Nationales Génie Côtier - Génie Civil \\ Cherbourg, 12-14 juin 2012}

\subsubsection{Stabilité au séisme.}

\subsubsection{Accélération sismique}

Les ouvrages devront être conçus pour répondre aux deux niveaux de séismes suivants :

- Niveau i : L'ouvrage peut subir quelques dégâts mais sa fonctionnalité n'est pas atteinte. Les dégâts subis devraient être réparables et les opérations portuaires ne devraient pas être interrompues par l'ampleur des dégâts et la nécessité d'effectuer les réparations.

- Niveau ii : L'ouvrage peut subir des dégâts substantiels mais sa fonction ne devrait pas être affectée. Il devrait être possible de rétablir son entière fonctionnalité à long terme. Les opérations portuaires peuvent être suspendues par la nécessité d'effectuer les réparations.

\subsubsection{Evènements sismiques}

Les ouvrages seront conçus pour faire face aux évènements sismiques en prenant en considération, via les coefficients de site de l'AFPS90, la modification des mouvements du sol par le sol existant entre le substratum et les ouvrages projetés, et éventuellement les spectres AFPS90.

- Evènements de niveau i : Cet événement est considéré comme ayant une probabilité de dépassement de $10 \%$ sur une période de 50 ans. Il est considéré comme défini par une accélération horizontale maximale de $0,16 \mathrm{~g}$ au substratum.

- Evènements de niveau ii : On définit par ailleurs des séismes de niveau II, pour lesquels les ouvrages devront rester réparables :

- Séisme proche, d'accélération maximum au niveau du substratum $0,24 \mathrm{~g}$ associée à une magnitude de 4,7 (échelle de Richter)

- Séisme lointain, d'accélération maximum au niveau du substratum $0,093 \mathrm{~g}$ associée à une magnitude de 8,5 (échelle de Richter).

\subsubsection{Traficabilité de la dalle de circulation longeant les ouvrages de protection :}

- La largeur de la dalle de circulation est de $7 \mathrm{~m}$ libre de toute contrainte (pipes du poste vracs liquides, équipements protection cathodique...) à au moins $+4.50 \mathrm{~m} \mathrm{ZH}$. Cette largeur de circulation de $7 \mathrm{~m}$ est réservée :

- sur la dalle de roulement qui est positionnée sur les digues à talus (digue principale et digue secondaire);

- sur la dalle réalisée sur le remblai situé le long de la partie en caissons de la digue principale jusqu'au second poste pétrolier sur toute son emprise au niveau de la zone de mise à poste plus une distance de $20 \mathrm{~m}$;

- Sur les caissons au-delà du second poste pétrolier de la digue principale; 
- Trois zones de retournement d'un diamètre de $15 \mathrm{~m}$ sont prévues : aux musoirs des digues principale et secondaire et à une position intermédiaire le long de la digue principale.

2.3.6 Infrastructures pour les futures conduites hydrocarbures et équipements des postes pétroliers :

L'emprise maximale pour la prise en compte des dimensions du rack des conduites hydrocarbures de vracs liquides est de $2,7 \mathrm{~m}$. Cette largeur est réputée satisfaire les besoins du futur exploitant pour disposer les différents pipes, équipements, et les dispositifs de compensation des éventuelles dilatations.

Des dispositions au niveau du mur de couronnement sont à prévoir pour la protection des conduites et de leurs supports pour des houles centennales extrêmes (90\%).

\subsection{Les terre-pleins}

2.4.1 Surface, cote d'arase et pente pour le drainage des eaux pluviales :

La surface de terre-pleins à l'arrière de la magistrale est celle obtenue jusqu'à l'intersection avec le relief en falaise existant à l'arrière de ces terre-pleins ou la digue principale du port Roulier existant. Ceci conduit à la surface de terre-plein derrière le quai à conteneurs de 150 ha.

Le remblaiement derrière le fond de darse ouest sera réalisé jusqu'à l'intersection de la falaise arrière. La surface correspondante devra être d'environ 5.4 ha.

La cote d'arase des terre-pleins du quai à conteneurs est de $+4,50 \mathrm{~m} \mathrm{ZH}$ au niveau du quai à conteneurs et aura pour le remblai une pente ascendante de $0,8 \%$ coté terre jusqu'à la limite sud. La côte d'arase des terre-pleins le long du cavalier Ouest de D1 est de $+4.50 \mathrm{~m}$ ZH et aura une pente ascendante de $0,8 \%$.

La cote d'arase des terre-pleins derrière le quai de service provisoire est d'au moins $+2,50 \mathrm{~m} \mathrm{ZH}$ avec un talus de raccordement entre ce terre-plein et le terre-plein du quai à conteneurs.

2.4.2 Compacité des terre-pleins (densité relative, résistance de pointe minimale mesurée au CPT, etc.) :

Les remblais des terre-pleins (hors matériaux adossé aux caissons de la digue principale et le terre-plein à l'arrière du quai de service provisoire) peuvent être scindés en deux catégories en fonction des possibilités techniques de mise en œuvre et de compactage :

- Catégorie 1 "Remblais sous l'eau" : ces remblais seront mis en œuvre par clapage, refoulement ou bennage à l'avancement.

- Catégorie 2 "Remblais hors d'eau" : cette partie de remblais pourra être mise en œuvre par les moyens terrestres et compactée par des moyens classiques de compactage. 


\section{XII ${ }^{\text {èmes }}$ Journées Nationales Génie Côtier - Génie Civil \\ Cherbourg, 12-14 juin 2012}

\subsubsection{Critères relatifs aux remblais Catégorie 1 :}

Dans le cas où les matériaux des remblais sont issus de dragage/déroctage ou d'extractions de sable au large, les matériaux et les remblais devront répondre aux critères suivants :

- D10>0.08 mm.

- Dmax $=100 \mathrm{~mm}$.

- Teneur en poids maximale de $6 \%$ de fines $(<80 \mu \mathrm{m})$.

- Une densité relative de l'ordre de $75 \%$ à $80 \%$.

Une valeur minimale de résistance à la pénétration de $10 \mathrm{MPa}$ sur toute la hauteur du remblai à partir de $2 \mathrm{~m}$ de profondeur (au-dessus, le matériau devra être compacté par la méthode classique). Cet objectif sera vérifié par des essais CPT.

Dans le cas où les matériaux des remblais sous l'eau sont issus des carrières terrestres, ils devront répondre aux critères suivants :

- Matière organique : $0 \%$.

- Courbe granulométrique étalée à agréer par le maître d'ouvrage.

- D6 $>0.08 \mathrm{~mm}$.

- Indice de Plasticité maximal de 10.

- Dmax $=300 \mathrm{~mm}$.

- Une densité relative de l'ordre de $75 \%$ à $80 \%$.

\subsubsection{Critères relatifs aux remblais Catégorie 2}

Les remblais terrestres de catégorie 2 doivent répondre aux critères suivants :

Remblai Type I - Remblais de Surface - dernier demi mètre des terre-pleins :

- Matière organique : $0 \%$.

- Analyse granulométrique : la granulométrie sera étendue.

- Dmax $=150 \mathrm{~mm}$.

- D10>0.08 mm.

- Limite d'Atterberg : limite de liquidité inférieure à 50, indice de plasticité $<10$.

- Essai Proctor : compaction à $98 \%$ du Proctor modifié.

- Une valeur minimale de CBR de $10 \%$ et un EV2>100 MPa (essais de plaques) avec le rapport $(E V 2 / E V 1)<2$ sont exigés pour les $50 \mathrm{~cm}$ supérieurs du remblai type I.

Remblai Type II - couches en dessous du Remblai de Surface

- Matière organique maximum de $2 \%$.

- Dmax $=250 \mathrm{~mm}$.

- Indice de plasticité inférieure à 20.

- Essai Proctor : compaction à $95 \%$ du Proctor modifié.

\subsubsection{Tassements admissibles}

Les tassements admissibles maximaux au niveau de la surface réceptionnée seront inférieurs aux valeurs suivantes : 
- $75 \mathrm{~mm}$ au bout de $1 \mathrm{an}$, et le cas échéant, $100 \mathrm{~mm}$ en deux ans si ce $1^{\mathrm{er}}$ critère ne peut être respecté.

- $125 \mathrm{~mm}$ au bout de 5 ans.

- $150 \mathrm{~mm}$ au bout de 20 ans.

Ces critères s'appliquent sur la totalité de la plate forme y compris dans la zone proche du quai entre les voies de portiques.

Les tassements différentiels devront rester inférieurs à $0,5 \%$ en toutes directions soit un différentiel de tassement de $50 \mathrm{~mm}$ sur $10 \mathrm{~m}$.

Les tassements seront calculés sur la base d'un modèle mathématique, qui tient compte notamment de la plasticité du sol de fondation en place. Les charges de long terme nécessaires pour effectuer ces calculs sont comme suit. Elles doivent être appliquées simultanément au toit de la surface des terre-pleins :

- Un mètre d'épaisseur de chaussée,

- Une charge équivalente de conteneurs : $40 \mathrm{kPa}$.

\section{Critères de durabilité des ouvrages}

Pour répondre à l'exigence de durabilité des ouvrages correspondant à une durée de vie minimale de 100 ans, les exigences suivantes ont été définies :

\subsection{Caractéristiques et composition des bétons armés du projet}

Les ouvrages en béton armé seront confectionnés avec un BHP. La composition de ce béton sera basée sur les principes suivants :

- Liant total minimum de $420 \mathrm{~kg} / \mathrm{m}^{3}$.

- Résistance à la compression nominale à $28 \mathrm{j}$ supérieure à $40 \mathrm{MPa}$.

- Résistance à la traction nominale à $28 \mathrm{j}$ supérieure à $3 \mathrm{MPa}$.

- Ciment utilisé du type Prise Mer aux cendres volantes.

- Rapport en poids eau efficace/liant total inférieur à 0.35

- Chaque arrivage sur le chantier des produits incorporés dans les bétons, devra être accompagné du bon de livraison correspondant, contenant notamment les indications relatives à la conformité à la norme concernée.

- Au moins pour les voiles, le gradient thermique des bétons mis en œuvre ne devra pas dépasser $20^{\circ} \mathrm{C}$ entre la surface et le cœur du béton. Pour les autres parties, ce principe reste valable, sauf justification de non préjudice pour les ouvrages fournie par l'Entrepreneur.

- Le coefficient de diffusion des chlorures à 90 jours sera au maximum $1,5 \times 10^{-12} \mathrm{~m}^{2} / \mathrm{s}$ pour la partie située au-dessus de -4hydro et de $5 \times 10^{-12} \mathrm{~m}^{2} / \mathrm{s}$ pour la partie au-dessous de $-4 \mathrm{~m} \mathrm{ZH}$. Toutefois, pour la partie située au-dessus de -4hydro, des valeurs au-delà de $1,5 \times 10^{-12} \mathrm{~m}^{2} / \mathrm{s}$ sont acceptables tant qu'elles restent inférieures à $2,5 \times 10^{-12} \mathrm{~m}^{2} / \mathrm{s}$ et dans la mesure où $95 \%$ des résultats consécutifs individuels sont inférieurs ou égaux à $1,5 \times 10^{-12} \mathrm{~m}^{2} / \mathrm{s}$. Les essais sont réalisés exclusivement sur des éprouvettes de 


\section{XII ${ }^{\text {èmes }}$ Journées Nationales Génie Côtier - Génie Civil \\ Cherbourg, 12-14 juin 2012}

laboratoire. La mesure du coefficient de diffusion sera effectuée selon la procédure NTB 492.

- L'épaisseur d'enrobage comptée à partir du nu des aciers est fixée à $80 \mathrm{~mm}$ pour toutes les faces extérieures et à $70 \mathrm{~mm}$ pour les faces intérieures et pour les deux faces du radier.

- Pour se prémunir contre la pénétration à jeune âge des chlorures, La fumée de silice sera incorporée au béton au-dessus de la cote $-4 \mathrm{~m} \mathrm{ZH}$.

- La teneur en chlorures maximale pour le sable doit être inférieure à $0,06 \%$ et pour le gravier et le gravillon doit être inférieure à $0,03 \%$.

- Tous les bétons armés seront munis d'une protection cathodique.

\subsection{Caractéristiques des bétons non armés}

- Liant total minimum de $335 \mathrm{~kg} / \mathrm{m}^{3}$.

- Résistance à la compression nominale à $28 \mathrm{j}$ supérieure à $30 \mathrm{MPa}$.

- Résistance à la traction nominale à $28 \mathrm{j}$ supérieure à $2,5 \mathrm{MPa}$.

- Ciment utilisé du type Prise Mer.

- Limitation de la température de mise en œuvre du béton à $30^{\circ} \mathrm{C}$ afin de limiter le retrait et de se prémunir contre les réactions sulfatiques internes.

\section{Normes et documents de référence}

Les normes qui serviront de référence sont les normes marocaines. En cas de non existence, les normes étrangères seront retenues dans l'ordre de prévalence suivant :

- Les normes Françaises.

- Les Eurocodes.

- British Standard.

- ASTM.

- AASHTO.

- D’autres normes équivalentes de référence internationale approuvées par le maître d'ouvrage.

Des documents qui serviront de référence sont :

- Fascicule 62 titre 1 section 2 du C.C.T.G. (BAEL 91) révisé 99.

- Recommandations AFPS 90 pour la rédaction des règles relatives aux ouvrages et installations à réaliser dans les régions sujettes aux séismes.

- "Méthodes pour la mesure des grandeurs associées à la durabilité", AFPC-AFREM, Journées techniques Durabilité des bétons, 11-12 décembre 1997, Toulouse France.

- Recommandations pour la prévention des désordres dus à l'alcali-réaction, LCPC juin 1994.

- Recommandations ROSA 2000 pour le Calcul aux Etats Limites des Ouvrages Maritimes et Fluviaux (pour les quais).

- DTU - CM66 : Règles de calcul des constructions en acier/additif, 1980. 
- Régies Neige et Vent 65 révisées 1984 (NV 65).

- Recommandations AFPS90 de l'Association Française du Génie Parasismique.

- "Liquefaction resistance of soils", by T.L. YOUD \& I.M. IDRISS in Journal of Geotechnical and Geoenvironmental Engineering, April 2001.

- "Manual on the use of rocks in coastal and shoreline engineering". CIRIA Special publication 83 / CUR Report 154,1991.

- US Army Coastal Engineering Research Centre, "Shore Protection Manual" (SPM) / Coastal engineering manual (CEM).

- Les exigences de conception pour la fabrication, la manipulation et le positionnement des unités ACCROPODE ${ }^{\circledR}$ se conformeront à la version la plus récente de "Technical Specification for the Fabrication and Placing of ACCROPODE® Blocks" publié par SOGREAH dans sa version française.

- Les pieux forés : Recueil des règles de l'art : LCPC - SETRA, 1978.

- "Calcul des fondations superficielles et profondes" par R. FRANK, Presses de l'ENPC, 1999.

- FOND 72 : LCPC-SETRA 1972.

- Pour les défenses de quais : "Guidelines for the design of fender systems", PIANC report of working group $332002,70 \mathrm{p}$.

- "Guidelines for the design of slopes under open piled quay walls" ; PIANC report of Working Group 22 of the Permanent Technical Committee II: Supplement to PIANC bulletin $\mathrm{n}^{\circ}$ 96, 1997.

- "Input data of propeller induced velocities for dimensioning of bed protection near quay walls", by K. ROMISCH and W. HERING, PIANC bulletin $n^{\circ} 109$, 2002, pp 511.

- British standards: BS 6349 Parties 1 et 4, BS 5950, BS 6405, BS 1663, JIS G3105/BS 5400 Partie 6.

\section{Conclusion}

Le projet de construction du port Tanger Med 2 rentre dans le cadre du projet du complexe portuaire de Tanger Med avec le port Tanger Med 1 et les ports roulier et à passagers en cours d'exploitation et ses trois vastes zones franches d'une superficie globale de près de 1000 ha.

Il s'agit donc d'un projet d'une importance capitale pour lequel les standards techniques de qualité les plus contraignants ont été retenus pour la construction de ses ouvrages et pour la réussite de son exploitation.

\section{Référence bibliographique}

PIANC -MarCom Working Group 24- (1995). Criteria for movements of moored ships in harbours - a practical guide. Report of WG 24 PTC2, Supplement to bulletin $n^{\circ} 88$, Brussels, Belgium. 